\title{
Surface plasmons for nanofabrication
}

Pieter G. Kik, Stefan A. Maier, Harry A. Atwater

Pieter G. Kik, Stefan A. Maier, Harry A. Atwater, "Surface plasmons for nanofabrication," Proc. SPIE 5347, Micromachining Technology for MicroOptics and Nano-Optics II, (29 December 2003); doi: 10.1117/12.532613

EDIE Event: Micromachining and Microfabrication, 2004, San Jose, California, United States 


\title{
Surface plasmons for nanofabrication
}

\author{
Pieter G. Kik, ${ }^{*}$ Stefan A. Maier, and Harry A. Atwater \\ Thomas J. Watson Laboratory of Applied Physics, California Institute of Technology, \\ Pasadena, CA 91125, USA
}

\begin{abstract}
The diffraction limit is the major stumbling block in pushing optical lithography to feature sizes smaller than $\sim 50 \mathrm{~nm}$. One approach to circumvent the diffraction limit in optical lithography has been to use optical near-field probes to perform local writing of resist layers. This approach suffers from low writing speeds due to the sequential nature of the process. We discuss two near-field optical illumination schemes that are compatible with broad-beam exposure and high throughput nanofabrication. The first approach concerns a method that can be used to print patterns with feature sizes below $50 \mathrm{~nm}$ using standard photoresist. The method relies on the plasmon resonance occurring in nanoscale metallic particles. Nanoparticle surface plasmons can be excited resonantly, producing a strongly enhanced dipole field around the particle. This enhanced near field can be used to locally expose a thin resist layer. Experiments and simulations show that feature sizes < 50nm can be produced using an exposure wavelength of $400 \mathrm{~nm}$. The second approach involves projecting near-field patterns using planar metal films. It has been predicted that thin metal films may be used to generate images with a spatial resolution better than the diffraction limit. We present simulations that reveal the role of surface plasmons in such near-field imaging with planar metal films.
\end{abstract}

Keywords: lithography, contact printing, surface plasmon, nanoparticles, pattern replication, near field, perfect lens

\section{INTRODUCTION}

As device dimensions in integrated circuits are getting smaller, it is becoming increasingly difficult to use conventional optical projection lithography in the fabrication process. This is due to the fact that minimum feature size in optical projection lithography is given by the diffraction limit, which is approximately half an optical wavelength. Further reduction of device dimensions consequently involves a reduction in illumination wavelength into the deep ultraviolet, ${ }^{1}$ the extreme ultraviolet (EUV), ${ }^{2}$ or the X-ray regime. ${ }^{3}$ This requires some radical changes in the lithographic process, including the development of new light sources, new photoresists, and reflective optics as opposed to conventional refractive optics.

An alternative to optical projection lithography is near-field optical lithography. Optical near fields are the localized fields that exist in the vicinity of polarized surfaces and interfaces. One example of near-field optical lithography is the use of the tip of a near-field scanning optical microscope to illuminate photoresist layers. In this case, light emitted from a nanoscale aperture in a metal-coated optical fiber is used to locally expose a photoresist layer. In this process the size of the aperture determines the achievable resolution. The major drawback of this approach lies in its inherently low throughput. This is due to two issues: 1 . the optical power that can be transmitted through a nanoscale aperture is quite low, typically less than a microwatt for a $100 \mathrm{~nm}$ diameter aperture, and 2. the exposure takes place in a sequential manner, i.e. the local illumination source needs to be scanned over the surface during the lithographic process. To solve these problems, one would need to construct a mask that consists of an array of nanoscale light sources. This can be achieved by generating a mask that contains arrays of metal nanoparticles. The nanoparticles act as local field concentrators thanks to the existence of surface plasmons: when a metallic nanoparticle is placed in an optical field, it can support collective electron oscillations known as surface plasmons. Associated with the oscillating charge on the particle is a localized oscillating dipole field near the particle. When the excitation occurs resonantly, i.e. when the excitation frequency coincides with the natural relaxation frequency of the particle, this can result a strongly enhanced electrical field near the particle, providing the high near-field intensity required for high throughput optical

\footnotetext{
* Present address: School of Optics/CREOL, University of Central Florida, Orlando, FL; e-mail: kik@ creol.ucf.edu
} 

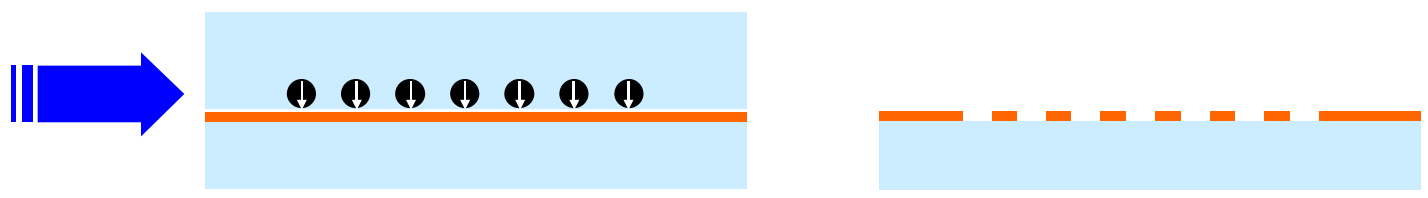

Figure 1 Schematic representation of plasmon printing, showing (a) glancing angle illumination using polarized visible light, producing enhanced resist exposure directly below the metal nanostructures in the mask layer, and (b) the resulting pattern in the resist layer after development.

nanolithography. In the first part of this article we discuss the process of nanoparticle-enhanced near-field lithography. In the second part we discuss how surface plasmons on planar metal layers can be used to obtain optical projection of subwavelength features over short distances.

\section{METAL NANOPARTICLE PLASMONS FOR NEAR-FIELD LITHOGRAPHY}

Figure 1 shows a schematic representation of the plasmon enhanced near-field printing process: a transparent mask containing metal particles near its surface is brought into intimate contact with a thin layer of photoresist. The resist covered substrate is then illuminated with p-polarized light at the plasmon resonance frequency of the particles. The resulting enhanced optical field around the metal particles will cause increased exposure of the resist layer directly below the particles. The exposure is interrupted when the exposure threshold is reached in the resist directly beneath the particles. Although the whole resist film has been illuminated, subsequent development of the resist will only affect the areas that received locally enhanced exposure. In this way a replica of the mask pattern is produced in the resist layer.

The choice of particle material depends on the required wavelength - the resonance wavelength should fall within the resist sensitivity. In order to be able to use standard g-line photoresist, the resonance wavelength should lie between $300 \mathrm{~nm}$ and $460 \mathrm{~nm}$. Additionally, to obtain high field enhancement, long electron scattering times are desirable. Silver is a good candidate, with long reported electron scattering times ${ }^{4}$ up to $10 \mathrm{fs}$ and a resonance occurring at a wavelength of $\sim 360 \mathrm{~nm}$ in air. The resonance frequency is not a fixed quantity: for a spherical particle, the surface plasmon resonance occurs at the wavelength $\lambda$ for which $\varepsilon_{\text {particle }}(\lambda)=-2 \varepsilon_{\text {matrix }}(\lambda)$ with $\varepsilon_{\text {particle }}$ and $\varepsilon_{\text {matrix }}$ the real part of the dielectric functions of the particle and the surrounding material respectively. Consequently, the wavelength at which resonance occurs can be tuned by changing the refractive index of the surrounding material.

For high field enhancement, the phase of the optical field should be approximately constant over the particle volume. When large particles are used, the field enhancement will drop due to the excitation of multipolar oscillations in the particles. In practice this means that the particles should have a diameter on the order of $0.1 \lambda$ or smaller, with $\lambda$ the illumination wavelength. For particles in this size range, electron relaxation due to surface scattering becomes significant. This surface scattering becomes more important for smaller particle diameters, and adversely affects the field enhancement. In this article, we therefore focus on particles with a diameter of $\sim 0.1 \lambda$.

The details of the plasmon printing process depend on the magnitude of the field enhancement effect and the exact spatial field distribution around the metal nanoparticles. Field distributions during illumination were calculated using 3D calculations based on the Finite Integration Technique ${ }^{5}$ (FIT). The simulated geometry consists of a $40 \mathrm{~nm}$ diameter silver particle on a $50 \mathrm{~nm}$ thick resist layer (modeled as a purely dielectric layer with a refractive index $\mathrm{n}=1.7$ ) on glass $(n=1.4)$. The silver particle is embedded in an index matched medium $(n=1.4)$, which could for example be a conformable PDMS mask. The simulation volume contains $\sim 4 \times 10^{6}$ mesh points with a graded mesh density to obtain a high mesh density around the silver particle (mesh spacing $2 \mathrm{~nm}$ ) while keeping the total number of mesh lines manageable. The particle is excited by a p-polarized plane wave coming in at glancing angle (between $70^{\circ}$ to $90^{\circ}$ off the surface normal). The complex dielectric function $\varepsilon(\omega)$ of silver is approximated by a Drude model, given by 

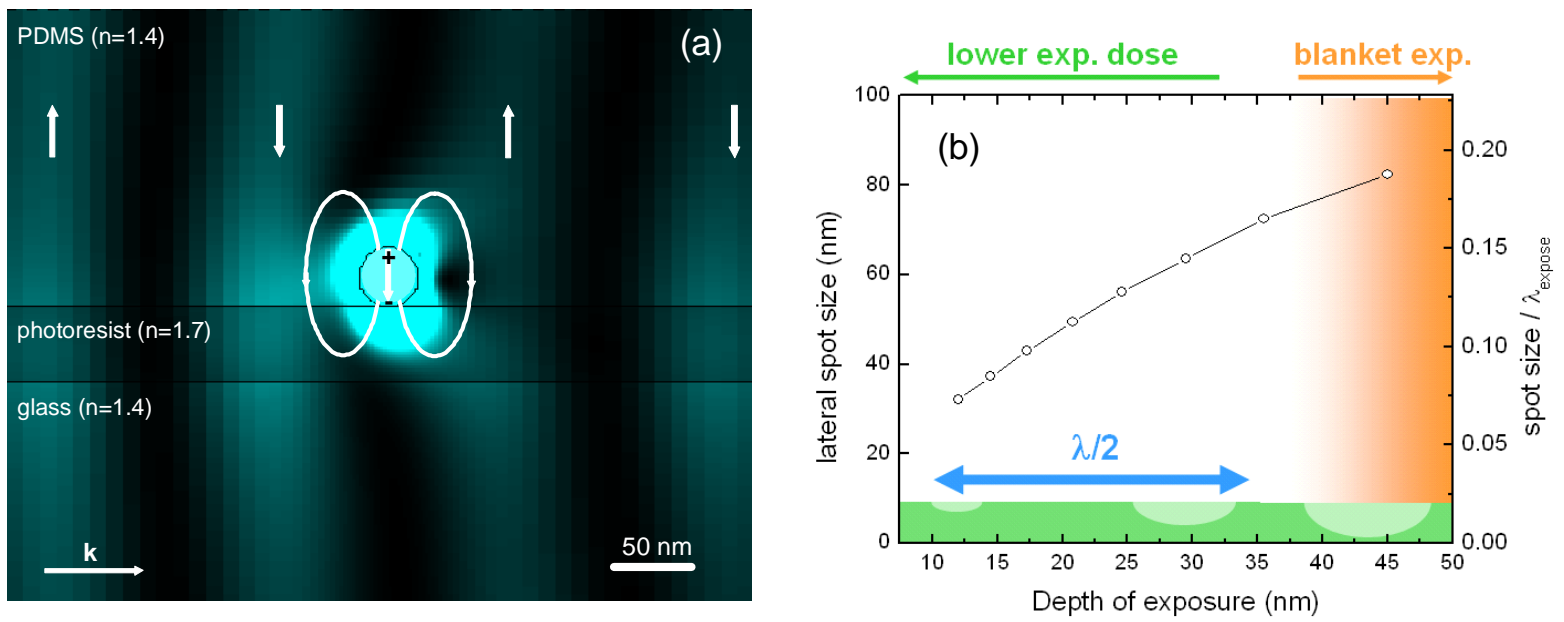

Figure 2 (a) Snapshot of the local energy density distribution around a $40 \mathrm{~nm}$ silver particle on photoresist under illumination with ppolarized light $(\lambda=439 \mathrm{~nm})$ at the surface plasmon resonance frequency, as obtained from 3D Finite Difference Time Domain simulations. The vertical arrows indicate the direction of the electric field in the illuminating wave. (b) The effect of exposure dose on feature width and exposure depth.

$$
\varepsilon(\omega)=1-\frac{\omega_{p}^{2}}{\omega^{2}-i \omega \gamma}
$$

with $\omega$ the angular frequency of the optical field, $\omega_{p}$ the bulk plasma angular frequency, and $\gamma$ the electron collision frequency defined as $\gamma=1 / \tau$ with $\tau$ the average time between subsequent electron collisions. The electron collision frequency is set to $10^{14} \mathrm{~s}^{-1}$ taken from Ref. 4 , corresponding to $\tau=10 \mathrm{fs}$. The value for $\omega_{p}$ is set to $1.08 \times 10^{16} \mathrm{rad} / \mathrm{s}$ in order to have the Drude dielectric function coincide with literature values for $\varepsilon(\omega)$ around $\lambda=430 \mathrm{~nm}$. The total length of the simulation is $30 \mathrm{fs}$ or 20 optical cycles, ensuring that the amplitude of the field oscillation in and around the particle has reached its steady state. The time step used in the simulations is two attoseconds or 1/750 of an optical cycle.

Figure 2(a) shows a snapshot of the calculated field energy density around the $40 \mathrm{~nm}$ silver particle under plane wave excitation at $439 \mathrm{~nm}$. The optical wave is traveling in the $+\mathrm{x}$ direction (incidence angle of $90^{\circ}$ relative to the surface normal). High color intensity in the plot corresponds to high local energy density. The vertical arrows indicate the direction of the electric field. The image shows the situation in which a positive wave front (field up) has just passed the particle. This wave front has induced a strong opposing field inside the particle with a corresponding dipole field around the particle, as indicated schematically by the curved field lines. The particle has a $\pi / 2$ phase lag compared to the exciting wave, which is a signature of resonant excitation. Note the enhanced energy density around the particle compared to the exciting wave in an area smaller than the diffraction limit. This snapshot gives a first indication of the spatial extent of the enhanced field. The exposure depth and width will depend on the exposure dose: a long exposure time will thus produce a deep and relatively wide depression in the resist, and at high doses the entire layer will reach the exposure threshold of the resist. Figure 2(b) shows how the width of the exposed spot (measured $\sim 10 \mathrm{~nm}$ beneath the surface) depends on the exposure depth based on the simulation results. Note that a lateral feature size of $0.1 \lambda$ can be achieved with an exposure depth of $17 \mathrm{~nm}$.

It should be noted that the concave shape of the exposed area and the small thickness of the resist layer make pattern transfer via a lift-off process difficult. Another practical issue is the spacing between mask and substrate. Due to the short range of the field enhancement, the separation between mask and photoresist should be small $(<10 \mathrm{~nm})$. To allow for such small separation over large areas, conformable masks may be required. Despite these restrictions, this technique still has the potential to produce sub-diffraction-limit patterns in a parallel fashion using visible light and standard photoresist. 

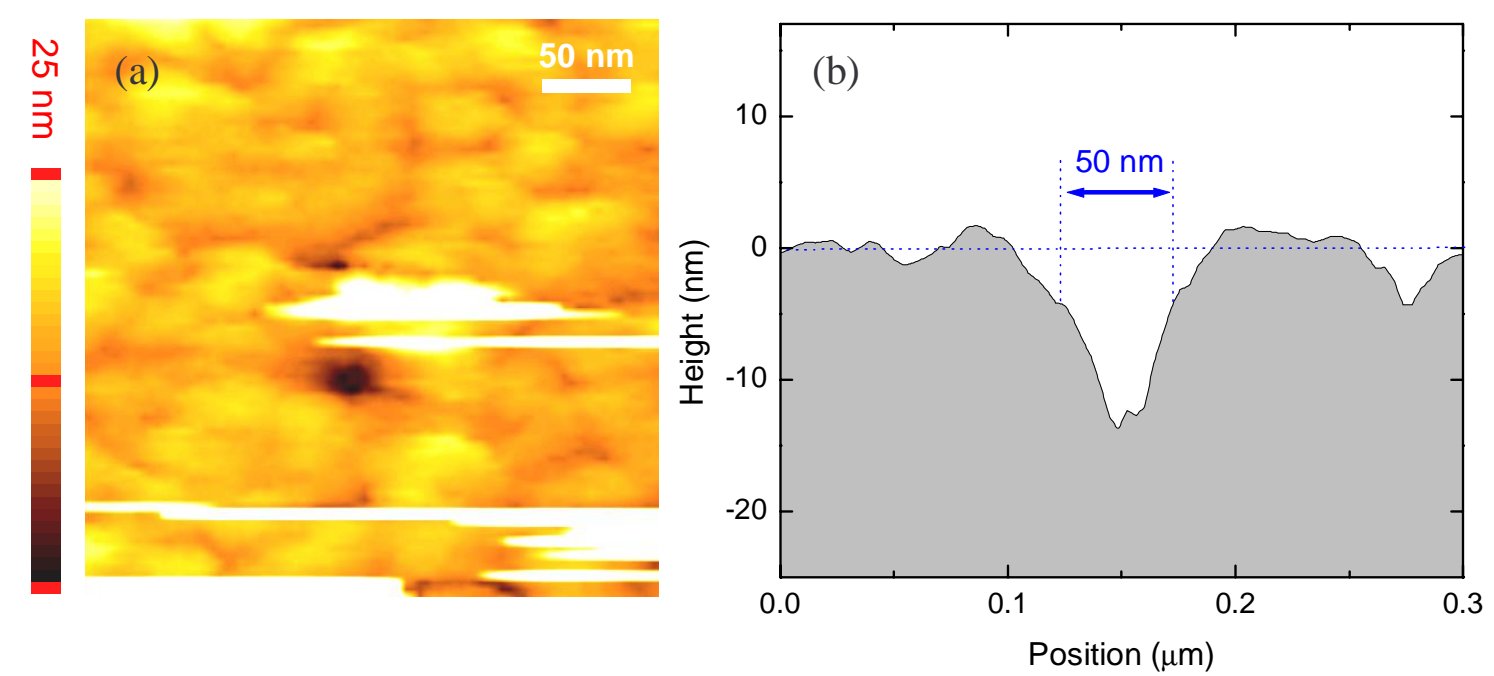

Figure 3 (a) Atomic Force Microscopy image of a 75 nm thick exposed and developed AZ1813 resist layer, showing the presence 41 $\mathrm{nm} \mathrm{Ag}$ particles on the surface (streaks) and a nanoscale depression attributed to locally enhanced resist exposure below a nanoparticle due to resonant excitation of a surface plasmon oscillation in the particle. (b) A cross-section through the imprint, showing a feature size of $50 \mathrm{~nm}(\sim 0.1 \lambda)$.

Our initial experiments have been aimed at obtaining a proof-of-principle. To avoid difficulties in achieving the desired nanoscale spacing between mask and resist layer, instead of using a conventional mask our experiments involve $41 \mathrm{~nm}$ diameter silver nanoparticles (the "mask") spray-deposited onto a thin resist layer. Glass substrates (surface roughness < $5 \AA$ RMS) were coated with standard g-line resist (AZ1813, Shipley), which has its maximum sensitivity in the wavelength range 300-450 $\mathrm{nm}$. The resist was diluted with AZ EBR dilutant (ratio AZ1813:EBR = 1:4) and subsequently spin coated onto the substrates at $5000 \mathrm{rpm}$ (60s) producing a smooth $\sim 75 \mathrm{~nm}$ thick film. The smoothness is important since in the experiments $\sim 40 \mathrm{~nm}$ diameter features need to be resolved. For illumination the output of a 1000 W Xe arc lamp was sent through a monochromator set to a wavelength of $410 \mathrm{~nm}$, and subsequently passed through a polarizer to obtain polarization normal to the sample surface. The beam was vertically compressed using a cylindrical lens to increase the power density, and sent to the sample at glancing incidence. The applied power densities were of the order of $1 \mathrm{~mW} / \mathrm{cm}^{2}$, and exposures times were in the range $10 \mathrm{~s}-300 \mathrm{~s}$. After exposure the films were developed for $20 \mathrm{~s}$ in developer (MF317), mixed with water in a 1:1 ratio to slow down development. Conventional exposure of the $75 \mathrm{~nm}$ thick resist layers showed normal development at these development conditions. The developed films were investigated using contact mode Atomic Force Microscopy.

The developed films were found to still have some residual nanoparticles on the surface. Using the AFM tip it was possible to move the particles over the surface, producing streaks in the AFM images. In addition to the particles, nanoscale dips were observed in the resist film, suggesting enhanced exposure in sub-wavelength size areas. Figure 3(a) shows an AFM scan of a $300 \times 300 \mu \mathrm{m}$ area, showing an approximately circular depression in the resist layer. Figure 3(b) shows a cross-section through the depression, showing a lateral size of approximately $50 \mathrm{~nm}$, and a depth of $12 \mathrm{~nm}$, possibly limited by the AFM tip shape. It should be noted that the identification of these nanoscale imprints is not fully unambiguous due to the relatively large resist roughness after development. Experiments are underway to further investigate the effect of plasmon enhanced resist exposure using e-beam defined masks containing a wide range of nanoparticle arrays.

\section{SURFACE PLASMONS FOR SUB-DIFFRACTION LIMIT OPTICAL PROJECTION}

As was mentioned in the introduction, the resolution limit for conventional optical projection lithography is given by the diffraction limit. A recent publication ${ }^{6}$ discussed the possibility of the projection of optical patterns with a resolution smaller than the diffraction limit over short distances. This would be possible by making use of the special properties of 

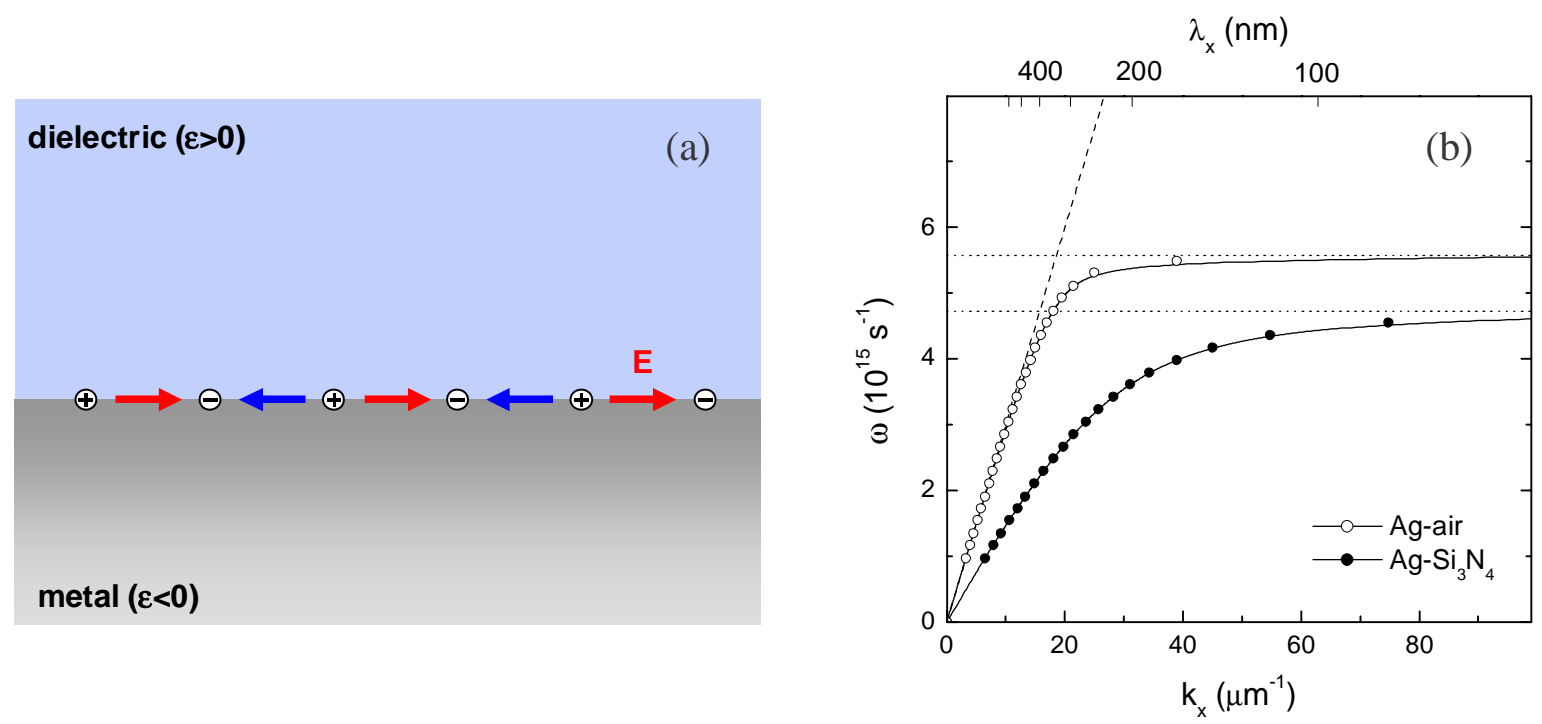

Figure 4 (a) Schematic representation of the charge distribution at a metal surface associated with the presence of a surface plasmon (b) Surface plasmon dispersion relation of a silver-air interface and a silver- $\mathrm{Si}_{3} \mathrm{~N}_{4}$ interface. The solid lines are guides to the eye. The dashed line represents the dispersion of light in air.

surface plasmons occurring at planar metal surfaces. In this section we discuss the role of surface plasmons in optical near-field projection of a nanoscale light source. This is done by performing three-dimensional (3D) simulations based on the Finite Integration Technique (FIT) using an accurate description of materials dispersion.

To understand the relevance of surface plasmons for near-field imaging, we first need to consider the surface plasmon (SP) dispersion relation of a flat metal surface. Surface plasmons at planar surfaces are longitudinal charge density fluctuations that occur at the boundary between a metal and a dielectric, see Fig. 4(a). The variations in charge density along the surface are described by a wavevector $k_{x}=2 \pi / \lambda_{x}$ with $\lambda_{x}$ the surface plasmon wavelength. The SP dispersion relation is given by ${ }^{7}$

$$
k_{x}(\omega)=\frac{\omega}{c} \sqrt{\frac{\varepsilon_{m}(\omega) \varepsilon_{d}(\omega)}{\varepsilon_{m}(\omega)+\varepsilon_{d}(\omega)}}
$$

with $\omega$ the angular frequency of the plasmon oscillation, $c$ the speed of light in vacuum, and $\varepsilon_{m}$ and $\varepsilon_{d}$ the real part of the dielectric functions of the metal and the surrounding medium respectively. The EM fields associated with surface plasmons drop off exponentially normal to the surface. Fig. 4(b) shows the dispersion relation of SPs on a silver (Ag) surface in air and in silicon nitride $\left(\mathrm{Si}_{3} \mathrm{~N}_{4}\right)$, respectively, calculated using Eq. 1 and literature values for the dielectric functions of silver ${ }^{8}$ and silicon nitride. ${ }^{9}$ The dielectric constant of air was taken to be 1 . The dashed line represents the dispersion relation of light in vacuum given by $\omega=c k_{x}$. For small values of $k_{x}$ the SP dispersion relation in air is lies close to the light line, but as $k_{x}$ increases, the dispersion relation asymptotically approaches $\omega=5.57 \times 10^{15} \mathrm{rad} / \mathrm{s}$ (indicated by the top dotted line) corresponding to a free-space wavelength of $\lambda_{f}=338 \mathrm{~nm}$. As can be seen in Fig. 4(b), this corresponds to the condition $\varepsilon_{l, A g}=-\varepsilon_{a i r}$. At this frequency, called the surface plasmon frequency $\omega_{\mathrm{SP}}$, the slope of the dispersion relation and thus the group velocity $v_{g}$ approaches zero, and the surface supports localized surface plasmons. For a silver surface in $\mathrm{Si}_{3} \mathrm{~N}_{4}$ the surface plasmon frequency is found to be $4.73 \times 10^{15} \mathrm{rad} / \mathrm{s}$ corresponding to a free-space wavelength of $\lambda_{f}=399 \mathrm{~nm}$. Figure 4(b) shows the necessary ingredients of near-field optical projection: at $\omega_{\mathrm{SP}}$ surface plasmons can provide charge distributions (and corresponding electric field distributions) with a wide range of high spatial frequencies, all oscillating at the same $\omega_{\text {SP. }}$ By exciting a coherent superposition of SP oscillations with different wavevectors on a metal surface it is thus theoretically possible to generate an image with a resolution far in excess of the diffraction limit. 


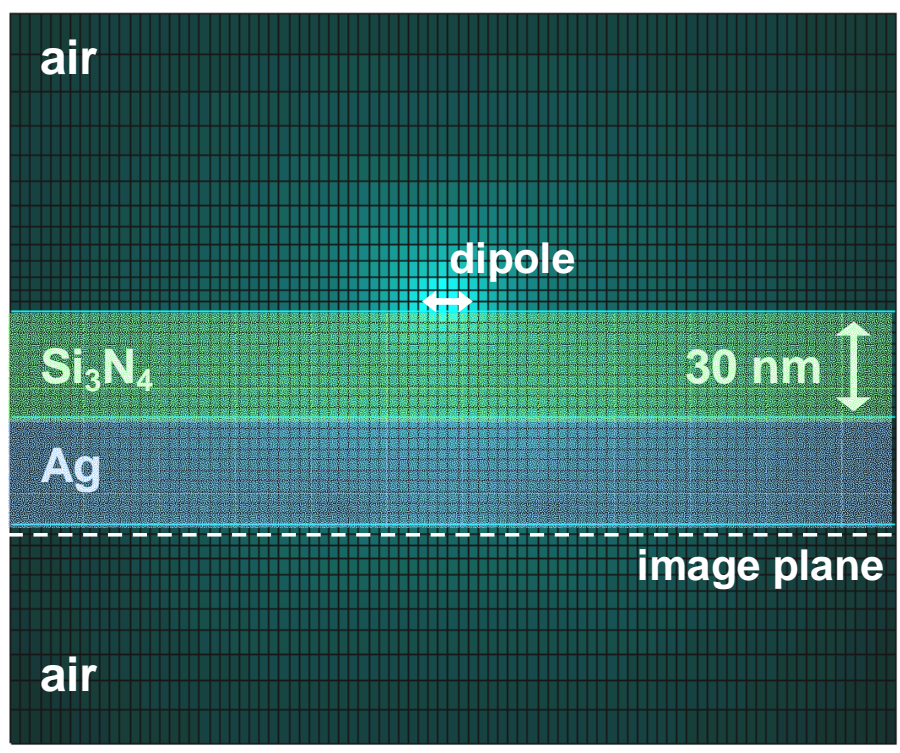

Figure 5. Central area of the 3D simulation volume, showing the $\mathrm{XZ}$ plane at $\mathrm{y}=0$. Note that the grid spacing is varied continuously to obtain high grid density at the metal-dielectric interface.

We investigate projection of a nanoscale light source represented by a point-dipole using a $30 \mathrm{~nm}$ thick Ag film supported on a $30 \mathrm{~nm}$ free-standing film of $\mathrm{Si}_{3} \mathrm{~N}_{4}$. The simulation geometry is shown in Fig. 5. We choose this example because it represents a situation that can be achieved experimentally by depositing silver onto silicon nitride membranes. Simulations are performed using a well established FIT based time domain code. The simulation volume spans $(500 \mathrm{~nm})^{3}$ and contains $2 \times 10^{6}$ grid points. The grid was graded along the z-axis (normal to the film surface) resulting in a minimum cell size of $(3 \mathrm{~nm})^{3}$ at the film location. The source is represented by an oscillating $\mathrm{x}$ polarized dipole (parallel to the surface) that is placed $3 \mathrm{~nm}$ away from the silicon nitride surface. In this geometry, the parallel electric field components of the dipole are predicted to be imaged near the silver-air interface. We study three cases - one in which the time-dependent dipole strength is given by a Gaussian-modulated sine wave with a center frequency of $4.73 \times 10^{15} \mathrm{rad} / \mathrm{s}\left(\lambda_{j}=399 \mathrm{~nm}\right)$ corresponding to $\omega_{\mathrm{SP}}$ of the $\mathrm{Ag}-\mathrm{Si}_{3} \mathrm{~N}_{4}$ interface, and two with frequencies below $\omega_{\mathrm{SP}}$ at frequencies that correspond to free space wavelengths of $458 \mathrm{~nm}$ and $514 \mathrm{~nm}$ respectively. The pulse bandwidth, measured at full width at half maximum (FWHM) was chosen to be $10 \%$ of the center frequency. Note that because of the finite bandwidth of our excitation pulse, it is important to accurately model the dielectric function of all materials over an extended frequency range. The number of time steps per optical cycle was 300 , and the simulation was run for over 20 optical cycles, allowing the dipole to reach its maximum amplitude. Reducing the bandwidth by a factor two, increasing the simulation time by a factor 2 , reducing the time step by a factor 2 , and increasing the number of grid points by a factor of 5 did not significantly alter the simulation results.

Figure 6(a) shows a representative snapshot of the $\mathrm{x}$-component of the electric field $\mathrm{E}_{\mathrm{x}}$ in the XZ-plane near the end of the simulation ( $\mathrm{t} \approx 25 \mathrm{fs}$ ) for an excitation frequency of $4.73 \times 10^{15} \mathrm{rad} / \mathrm{s}$. This time corresponds to the maximum amplitude of the excitation signal. The color scale is linear, with dark corresponding to negative values of $\mathrm{E}_{\mathrm{x}}$ and light corresponding to positive values. The $\mathrm{x}$-polarized dipole is nearing zero magnitude, and at the same time a localized spatially varying electric field distribution is observed at the $\mathrm{Ag}-\mathrm{Si}_{3} \mathrm{~N}_{4}$ interface. This electric field distribution is indicative of an oscillating charge density distribution due to the presence of surface plasmons at the interface. The location of the corresponding charge density maxima and minima is indicated by the + and - signs respectively. Note that the charge distribution is not perfectly periodic, showing that surface plasmons with different values of $k_{x}$ are simultaneously present. Note that the electric field distribution is approximately symmetric around the interface, an indication that at this frequency the values for $\varepsilon$ are indeed opposite on either side of the interface. ${ }^{7}$ Finally, note that the SP field strength in air is small on either side of the film stack, and consequently the surface 

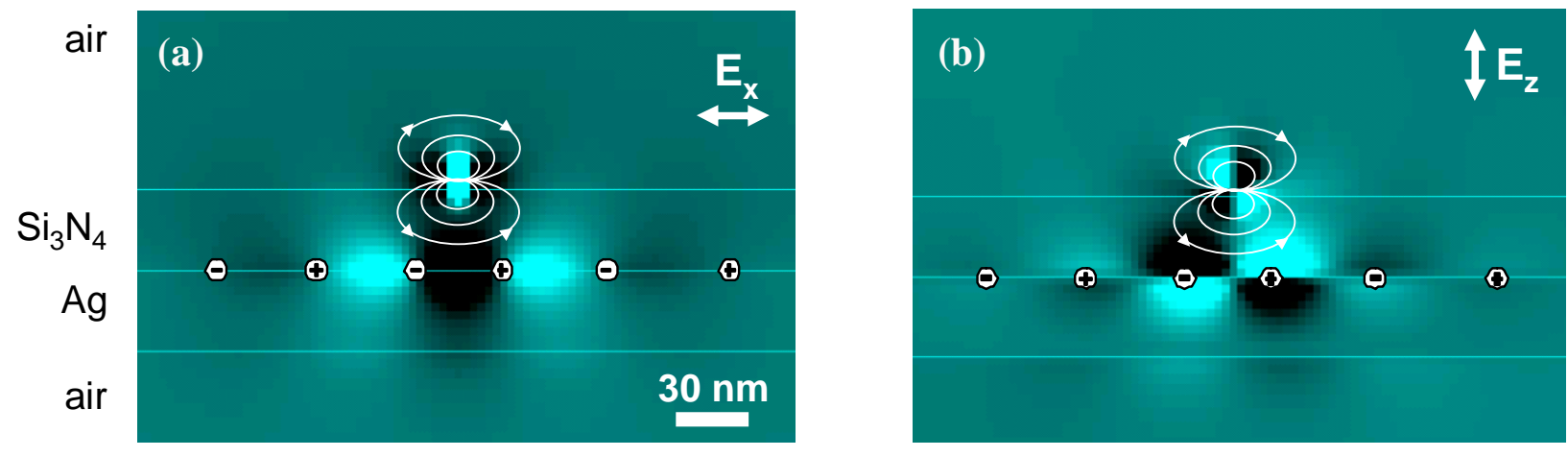

Figure 6. Snapshots of the electric field distribution near a planar metal film excited with an oscillating dipole at $\omega=4.73 \times 10^{15}$ $\mathrm{rad} / \mathrm{s}\left(\lambda_{\mathrm{f}}=399 \mathrm{~nm}\right)$ corresponding to the surface plasmon frequency, showing (a) the lateral component of the electric field $\left(\mathrm{E}_{\mathrm{x}}\right)$ and (b) the normal component of the electric field $\left(E_{z}\right)$ at $t \approx 25$ fs. The surface plasmon field distribution that is observed at the interface allows for near-field imaging.

plasmon dispersion relation is expected to be only weakly perturbed by the finite thickness of the films at these frequencies. Figure 6(b) shows the corresponding distribution of the $\mathrm{Z}$-component of the electric field ( $\mathrm{E}_{\mathrm{z}}$ ). As expected, $\mathrm{E}_{\mathrm{z}}$ peaks near maxima and minima of the charge distribution.

Figure 7(a) again shows the $\mathrm{Ag}-\mathrm{Si}_{3} \mathrm{~N}_{4}$ surface plasmon dispersion relation, indicating the two excitation frequencies below $\omega_{\text {SP. }}$. Fig $7(\mathrm{~b})$ shows the time averaged distribution of the lateral field strength in the image plane obtained at these excitation frequencies, measured at a distance of $3 \mathrm{~nm}$ (equal to one grid spacing) from the Ag surface. The black line represents a reference simulation in which the $\mathrm{Ag}-\mathrm{Si}_{3} \mathrm{~N}_{4}$ bilayer has been replaced by a $60 \mathrm{~nm}$ thick film of silicon nitride. We see that local illumination at frequencies below $\omega_{\text {SP }}$ leads to the excitation of standing waves with a well defined $\mathrm{k}_{\mathrm{x}}$, as expected from Fig. 7(a). Note that the field distribution in the image plane is in fact broadened compared to the case where no Ag layer is present. Fig 8(a) and 8(b) show the corresponding graphs for
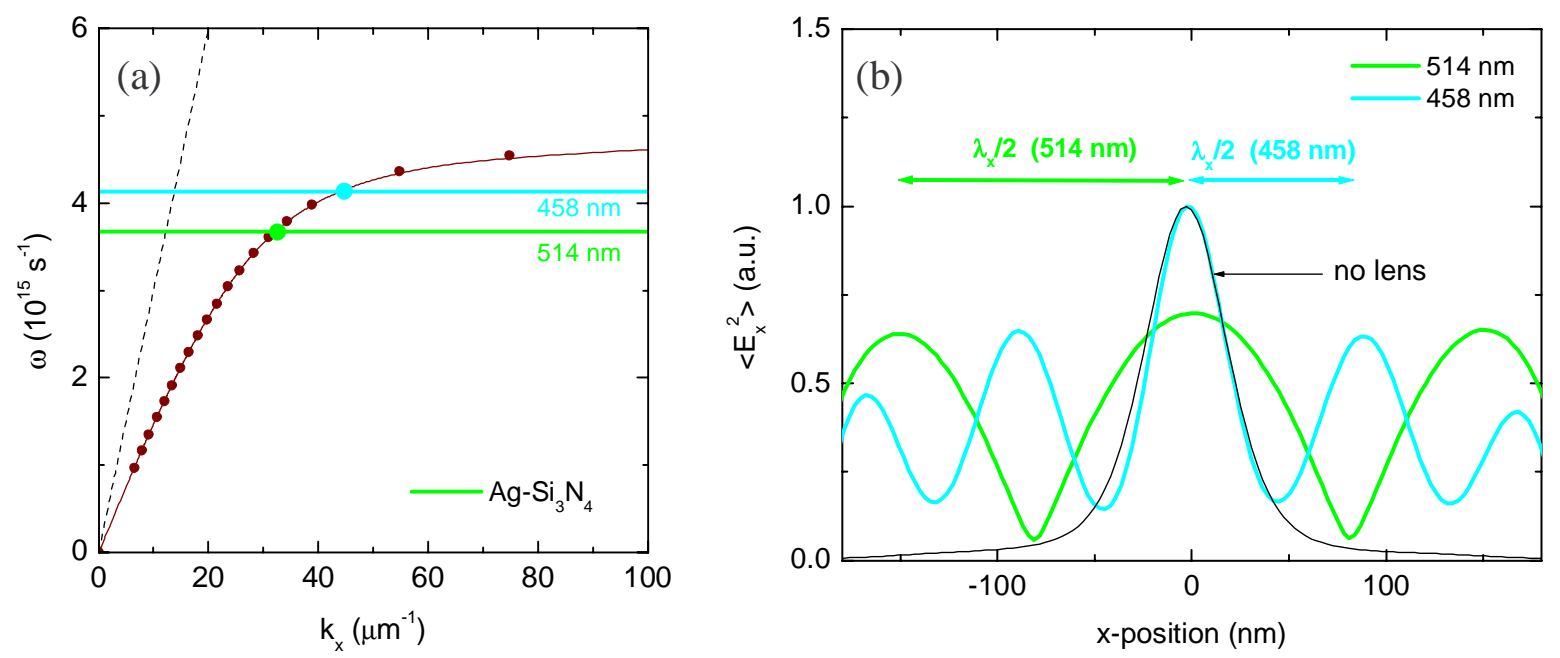

Figure 7. (a) Surface plasmon dispersion relation of a silver- $\mathrm{Si}_{3} \mathrm{~N}_{4}$ interface indicating to excitation wavelengths below $\omega_{\mathrm{SP}}$. (b) Time averaged field distributions in the image plane of the imaging structure shown in Fig. 5 for an excitation frequencies of $4.12 \times 10^{15}$ $\mathrm{rad} / \mathrm{s}$ and $3.67 \times 10^{15} \mathrm{rad} / \mathrm{s}$ corresponding to free-space wavelengths of $458 \mathrm{~nm}$ and $514 \mathrm{~nm}$ respectively. Also shown is the obtained distribution for a case in which the metal-dielectric bilayer is replaced by a solid $\mathrm{Si}_{3} \mathrm{~N}_{4}$ layer, labeled "no lens". 

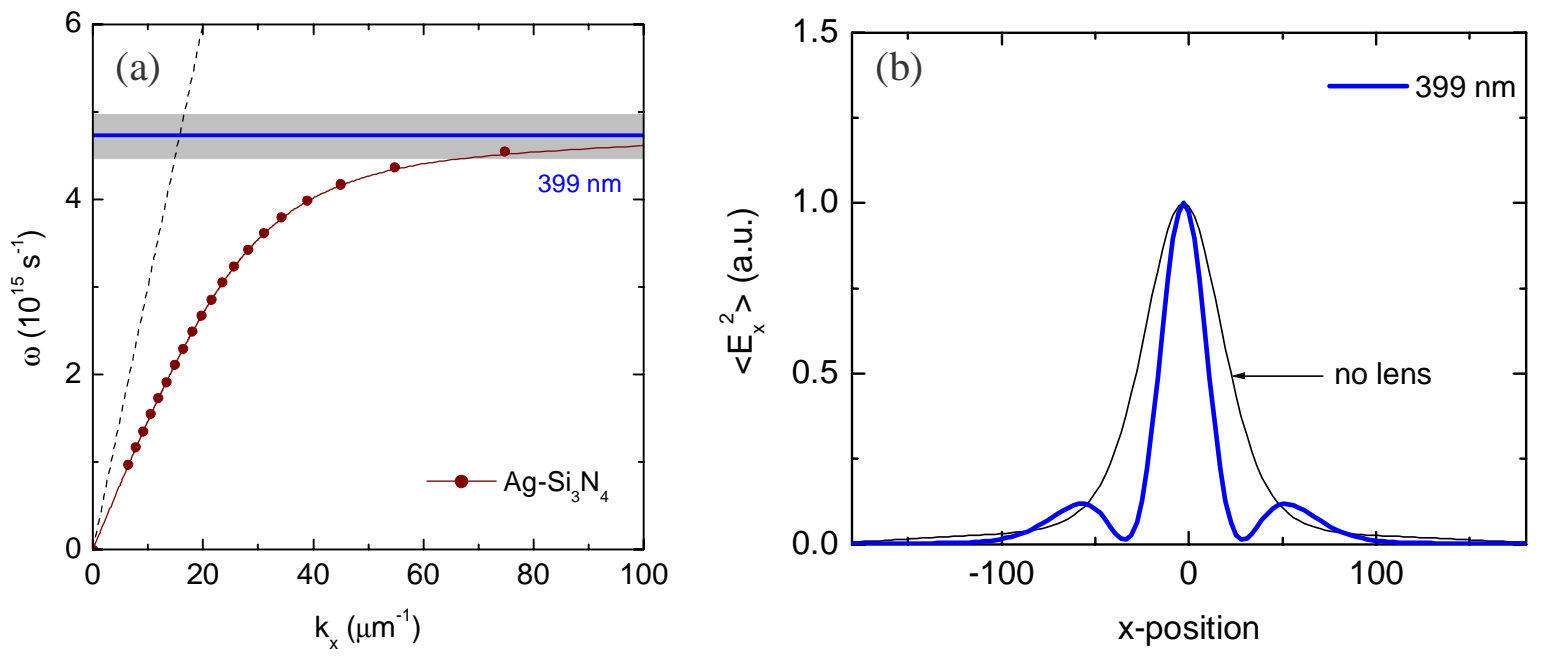

Figure 8. (a) Surface plasmon dispersion relation of a silver-Si $\mathrm{N}_{4}$ interface indicating excitation at $\omega_{\mathrm{SP}}$. (b) Time averaged field distribution in the image plane of the imaging structure shown in Fig. 5 for an excitation frequencies of $4.73 \times 10^{15} \mathrm{rad} / \mathrm{s}$ corresponding to a free-space wavelength of $399 \mathrm{~nm}$. Also shown is the obtained distribution for a case in which the metal-dielectric bilayer is replaced by a solid $\mathrm{Si}_{3} \mathrm{~N}_{4}$ layer, labeled "no lens".

excitation at $\omega_{\text {SP. }}$. We see that in this case the presence of the silver layer improves the resolution, reducing the FWHM of the field distribution in the image plane by a factor 2 from $51 \mathrm{~nm}$ to $27 \mathrm{~nm}$. When looking at the intensity distribution rather than the distribution of $E_{x}$, the focusing effect is slightly less pronounced, giving a resolution enhancement of a factor 1.4.

\section{CONCLUSIONS}

We have shown that the local field enhancement occurring around metal nanoparticles when they are excited at the surface plasmon resonance frequency can be used to print nanoscale features in thin resist layers. Feature sizes below $\lambda / 10$ can be generated in a parallel fashion using visible illumination and standard g-line photoresist. Additionally, we have shown computationally that optical projection at sub-diffraction limit resolution is possible by utilizing surface plasmons generated at a metal-dielectric interface. This work is supported by the Air Force Office of Scientific Research.

\section{ACKNOWLEDGEMENTS}

This work was supported by the National Science Foundation and the Air Force Office of Scientific Research.

\section{REFERENCES}

\footnotetext{
${ }^{1}$ M. Rothschild, T. M. Bloomstein, J. E. Curtin, D. K. Downs, T. H. Fedynyshyn, D. E. Hardy, R. R. Kunz, V. Liberman, J. H. C. Sedlacek, R. S. Uttaro, A. K. Bates, and C. Van Peski, J. Vac. Sci. Technol. B 17, 3262 (1999)

${ }^{2}$ C. W. Gwyn, R. Stulen, D. Sweeney, and D. Attwood, J. Vac. Sci. Technol. B 16, 3142 (1998)

${ }^{3}$ J. P. Silverman, J. Vac. Sci. Technol. B 16, 3137 (1998)
} 
${ }^{4}$ B. Lamprecht, A. Leitner, and F. R. Aussenegg, Appl. Phys. B 64, 269 (1997)

${ }^{5}$ S. Gutschling, H. Kruger, T. Weiland, Int. J. Num. Model. 13, 329 (2000)

${ }^{6}$ J. B. Pendry, Phys. Rev. Lett. 85, 3966 (2000)

${ }^{7}$ H. Raether, "Surface plasmons", (Springer-Verlag, 1988)

${ }^{8}$ P.B. Johnson, and R.W. Christy, Phys. Rev. B 6, 4370 (1972)

${ }^{9}$ E.D. Palik, "Handbook of optical constants", London, Academic Press (1985) 